PROCEEDINGS OF THE

AMERICAN MATHEMATICAL SOCIETY

Volume 130, Number 2, Pages 595-598

S 0002-9939(01)06061-0

Article electronically published on June 6, 2001

\title{
VIETORIS-BEGLE THEOREM FOR SPECTRAL PRO-HOMOLOGY
}

\author{
TAKAHISA MIYATA AND TADASHI WATANABE
}

(Communicated by Ronald A. Fintushel)

\begin{abstract}
Dydak and Kozlowski (1991) obtained a generalization of the Vietoris-Begle theorem for the cohomology theories induced by CW spectra. In this note we prove a dual of their theorem involving the pro-homology theories induced by CW spectra.
\end{abstract}

Given any $\mathrm{CW}$ spectrum $E$, let $E^{*}$ and $E_{*}$ denote the cohomology and homology theories induced by $E$ on the homotopy category $\mathbf{H C W}_{\text {spec }}$ of CW spectra. So $E^{*}$ and $E_{*}$ are contravariant and covariant functors from $\mathbf{H C W}$ spec to the category $\mathbf{A} \mathbf{b}_{*}$ of graded abelian groups. These functors extend to the functors pro- $E^{*}$ and pro $-E_{*}$ from the homotopy category HTop of pointed topological spaces to the pro-category pro- $\mathbf{A} \mathbf{b}_{*}$ of graded abelian groups. Let $\check{E}^{*}$ denote the Čech extension of $E^{*}$ over HTop.

Throughout the paper, every topological space $X$ is assumed to be unpointed and is regarded as the pointed space $X^{+}$with a discrete base point.

First, recall the following generalization of the Vietoris-Begle theorem:

Theorem 1 (Dydak and Kozlowski $[\mathrm{D}-\mathrm{K}]$ ). Let $E$ be a $C W$ spectrum, and let $f$ : $X \rightarrow Y$ be a closed surjective map between paracompact Hausdorff spaces such that Ind $Y=m<\infty$. If $\check{E}^{k}\left(f_{y}\right): \check{E}^{k}(y) \rightarrow \check{E}^{k}\left(f^{-1}(y)\right)$ is an isomorphism for all $y \in Y$ and $k=m_{0}, m_{0}+1, \ldots, m_{0}+m$, then $\check{E}^{k}(f): \check{E}^{k}(Y) \rightarrow \check{E}^{k}(X)$ is an isomorphism for $k=m_{0}+m$ and a monomorphism for $k=m_{0}+m+1$.

Here Ind $Y$ means the large inductive dimension of $Y$, and $f_{y}$ is the restricted $\operatorname{map} f \mid f^{-1}(y): f^{-1}(y) \rightarrow\{y\}$.

In this note we prove the following dual form:

Theorem A. Let $E$ be a ring spectrum, and let $f: X \rightarrow Y$ be a surjective map from a compact metric space $X$ to a compact metric space $Y$ with a finite covering dimension such that for each $y \in Y, f^{-1}(y)$ has a finite stable shape dimension. If pro $-E_{*}\left(f_{y}\right)$ : pro $-E_{*}\left(f^{-1}(y)\right) \rightarrow$ pro $-E_{*}(y)$ is an isomorphism for each $y \in Y$, then the induced morphism pro $-E_{*}(f)$ : pro $-E_{*}(X) \rightarrow$ pro $-E_{*}(Y)$ is an isomorphism.

Let $\Sigma$ denote the obvious functor from the homotopy category HCW of CW complexes to $\mathbf{H C W}_{\text {spec }}$ which assigns to each CW complex $X$ the suspension spectrum $\Sigma(X)$. For each topological space $X$, the stable shape dimension $\operatorname{sd}_{\text {spec }} X \leq n$

Received by the editors October 4, 1999 and, in revised form, July 25, 2000.

1991 Mathematics Subject Classification. Primary 55N05; Secondary 55N20, 55 P55.

Key words and phrases. Vietoris-Begle theorem, CW spectrum, pro-homology, compact metric space. 
provided whenever $\boldsymbol{p}=\left(p_{\lambda}\right): X \rightarrow \boldsymbol{X}=\left(X_{\lambda}, p_{\lambda \lambda^{\prime}}, \Lambda\right)$ is an HCW-expansion of $X$, for each $\lambda \in \Lambda$ there exists $\lambda^{\prime} \geq \lambda$ such that $\Sigma\left(p_{\lambda \lambda^{\prime}}\right)$ factors in $\mathbf{H C W}$ spec through a $\mathrm{CW}$ spectrum $Z$ with a dimension at most $n$, where an expansion is in the sense of [Ma-S]. Note that $\operatorname{sd}_{\text {spec }} X \leq \operatorname{sd} X$ where sd $X$ is the shape dimension of $X$ (see [Ma-S]) and there is a strict inequality case (see [Mi-S]).

The main tools are the duality between compact metric spaces and CW spectra in generalized stable shape theory and the Whitehead theorem in the generalized stable shape category, which we quote below:

Lemma 2 (Miyata [Mi, Theorem 5.3]). For each compact metric space $X$, there exist a $C W$ spectrum $X^{*}$ and a natural isomorphism

$$
\check{E}^{n}(X) \cong E_{-n}\left(X^{*}\right) .
$$

Lemma 3 (Miyata and Segal [Mi-S, Lemma 4.9]). Let $n, k \in \mathbb{Z}$ with $k \leq n$, and let $\left(f_{\lambda}\right): \boldsymbol{X}=\left(X_{\lambda}, p_{\lambda \lambda^{\prime}}, \Lambda\right) \rightarrow \boldsymbol{Y}=\left(Y_{\lambda}, q_{\lambda \lambda^{\prime}}, \Lambda\right)$ be a level morphism of inverse systems in $\mathbf{H C W}_{\text {spec }}$, which is an n-equivalence. Then every $\lambda \in \Lambda$ admits $\lambda^{\prime} \geq \lambda$ such that the following two statements hold:

i) if $Z$ is a $C W$ spectrum with a dimension at most $n$, then every morphism $h: Z \rightarrow Y_{\lambda^{\prime}}$ in $\mathbf{H C W}_{\text {spec }}$ admits a morphism $k: Z \rightarrow X_{\lambda}$ such that $f_{\lambda} k=$ $q_{\lambda \lambda^{\prime}} h$;

ii) if $Z$ is a $C W$ spectrum with a dimension at most $n-1$ and $k_{1}, k_{2}: Z \rightarrow X_{\lambda^{\prime}}$ are morphisms in $\mathbf{H C W}$ spec such that $f_{\lambda^{\prime}} k_{1}=f_{\lambda^{\prime}} k_{2}$, then $q_{\lambda \lambda^{\prime}} k_{1}=q_{\lambda \lambda^{\prime}} k_{2}$.

Before we prove the theorem, we prove the following lemmas:

Lemma 4. Let $E$ be a ring spectrum, and let $X$ be a topological space such that $\operatorname{sd}_{\text {spec }} X \leq n$ for some $n<\infty$. Then, if pro $-E_{*}(X)$ is trivial, $\check{E}^{*}(X)$ is trivial.

Proof. Let the ring spectrum $E$ be equipped with the maps $i: S \rightarrow E$ and $\mu: E \wedge$ $E \rightarrow E$, where $S$ is the sphere spectrum. Suppose $\operatorname{sd}_{\text {spec }} X \leq n<\infty$, and let $\boldsymbol{p}=$ $\left(p_{\lambda}\right): X \rightarrow \boldsymbol{X}=\left(X_{\lambda}, p_{\lambda \lambda^{\prime}}, \Lambda\right)$ be an $\mathbf{H C W}$-expansion of $X$. Suppose pro- $E_{*}(X) \approx$ 0 . Then $0 \approx E_{*}(\Sigma(\boldsymbol{X}))=\pi_{*}(\Sigma(\boldsymbol{X}) \wedge E)=\left(\pi_{*}\left(\Sigma\left(X_{\lambda}\right) \wedge E\right), \pi_{*}\left(\Sigma\left(p_{\lambda \lambda^{\prime}}\right) \wedge 1_{E}\right), \Lambda\right)$. Let $\lambda \in \Lambda$, and take $\lambda^{\prime} \geq \lambda$ as in Lemma 3. Since $\operatorname{sd}_{\text {spec }} X \leq n$, there exist $\lambda^{\prime \prime} \geq \lambda^{\prime}$, a CW spectrum $Z$ with a dimension at most $n$ and morphisms $g: \Sigma\left(X_{\lambda^{\prime \prime}}\right) \rightarrow Z$ and $h: Z \rightarrow \Sigma\left(X_{\lambda^{\prime}}\right)$ in $\mathbf{H C W}_{\text {spec }}$ such that $\Sigma\left(p_{\lambda^{\prime} \lambda^{\prime \prime}}\right)=h g$. Then the composite

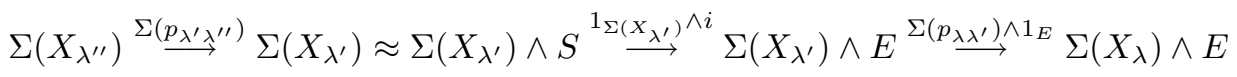

is trivial. For each element $\varphi_{\lambda}: \Sigma\left(X_{\lambda}\right) \rightarrow E$ of $E^{*}\left(\Sigma\left(X_{\lambda}\right)\right)$, there is a commutative diagram:

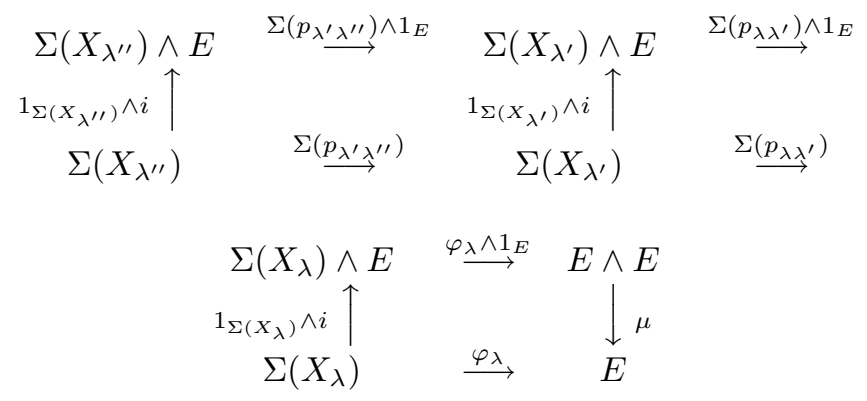


This implies that $E^{*}\left(\Sigma\left(p_{\lambda \lambda^{\prime}}\right)\right)\left(\varphi_{\lambda}\right)=0$. Hence $E^{*}\left(\Sigma\left(p_{\lambda \lambda^{\prime \prime}}\right)\right)=0$, which implies $\check{E}^{*}(X) \approx 0$.

Lemma 5. Let $E$ be a ring spectrum, and let $X$ be a compact metric space. Then, if $\check{E}^{*}(X)$ is trivial, pro $-E_{*}(X)$ is trivial.

Proof. Suppose $\check{E}^{*}(X) \approx 0$. Since $\check{E}^{*}(X) \approx E_{-*}\left(X^{*}\right)$ for some CW spectrum $X^{*}$ by Lemma 4 then $0 \approx E_{-*}\left(X^{*}\right) \approx \pi_{-*}\left(X^{*} \wedge E\right)$, which means that $X^{*} \wedge E$ is contractible. Let $\boldsymbol{p}=\left(p_{n}\right): X \rightarrow \boldsymbol{X}=\left(X_{n}, p_{n n+1}, \mathbb{N}\right)$ be an $\mathbf{H C W}$-expansion of $X$ such that each $X_{n}$ is a finite CW complex. Then this induces an $\mathbf{H C W} \mathbf{~ s p e c}^{-}$ coexpansion $\boldsymbol{p}^{*}=\left(p_{n}^{*}\right): \boldsymbol{X}^{*}=\left(X_{n}^{*}, p_{n n+1}^{*}, \mathbb{N}\right) \rightarrow X^{*}$ of $X^{*}$ in the sense of [Mi], where each $X_{n}^{*}$ is the Spanier-Whitehead dual of $\Sigma\left(X_{n}\right)$. So for each $n$, there is $m \geq n$ such that the composite

$$
X_{n}^{*} \approx X_{n}^{*} \wedge S \stackrel{1_{X_{n}^{*} \wedge i}^{\longrightarrow}}{\longrightarrow} X_{n}^{*} \wedge E \stackrel{p_{n m}^{*} \wedge 1_{E}}{\longrightarrow} X_{m}^{*} \wedge E
$$

is trivial. For each element $\varphi_{m}: X_{m}^{*} \rightarrow E$ of $E^{-*}\left(X_{m}^{*}\right)$, there is a commutative diagram:

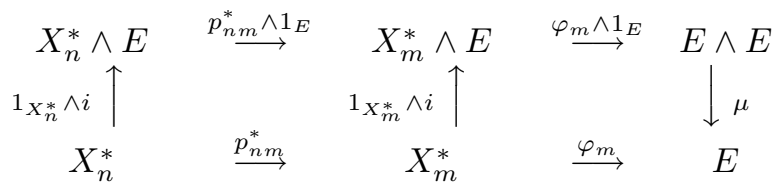

This implies that $E^{-*}\left(p_{n m}^{*}\right)\left(\varphi_{m}\right)=0$, and hence $E^{-*}\left(p_{n m}^{*}\right)=0$. Since $E_{*}(\Sigma(\boldsymbol{X})) \approx$ $E^{-*}\left(\boldsymbol{X}^{*}\right)=\left(E^{-*}\left(X_{n}^{*}\right), E^{-*}\left(p_{n n^{\prime}}^{*}\right), \mathbb{N}\right)$, then we have pro- $E_{*}(X) \approx 0$.

Lemma 6. Let $E$ be a ring spectrum, and let $f: X \rightarrow Y$ be a map of topological spaces. Then:

i) $\check{E}^{*}(f): \check{E}^{*}(Y) \rightarrow \check{E}^{*}(X)$ is an isomorphism iff $\check{E}^{*}\left(Y \cup_{f} C X\right)$ is trivial;

ii) pro $-E_{*}(f):$ pro $-E_{*}(X) \rightarrow$ pro $-E_{*}(Y)$ is an isomorphism iff pro $-E_{*}\left(Y \cup_{f}\right.$ $C X)$ is trivial.

Proof of Theorem A. Suppose pro- $E_{*}\left(f_{y}\right)$ is an isomorphism for each $y \in Y$. Then Lemmas 6 ii), 4 and 6 i) imply $\check{E}^{*}\left(f_{y}\right): \check{E}^{*}(y) \rightarrow \check{E}^{*}\left(f^{-1}(y)\right)$ is an isomorphism for each $y \in Y$. Since Ind $Y<\infty$, the Dydak and Kozlowski theorem implies that $\check{E}^{*}(f): \check{E}^{*}(Y) \rightarrow \check{E}^{*}(X)$ is an isomorphism. This fact, together with Lemmas 6 i), [5] and [6 ii), implies that pro- $E_{*}(f):$ pro $-E_{*}(X) \rightarrow$ pro- $E_{*}(Y)$ is an isomorphism.

A surjective map $f: X \rightarrow Y$ between compact metric spaces is said to be stably cell-like if each fibre has a trivial stable shape type (see Mi-S ). Theorem A with the spectrum $E$ being the sphere spectrum and [Mi-S, Theorem 6.1] imply

Corollary 7. Let $f: X \rightarrow Y$ be a stably cell-like map from a compact metric space $X$ with a finite stable shape dimension to a compact metric space $Y$ with a finite covering dimension. Then $f$ is an equivalence in the stable shape category.

Remark. The "figure eight like continuum" $X$ of Case and Chamberlin [C-C] has a trivial stable shape type but has a nontrivial shape type ([Ma] $)$. For any compact metric space $Y$, the natural projection $f: X \times Y \rightarrow Y$ is stably cell-like but not celllike. If $Y$ is finite dimensional, Corollary 7 then implies that $f$ is an equivalence in the stable shape category. The finite dimensionality of $Y$ in Theorem A is essential as pointed out in $[\mathrm{D}-\mathrm{K}]$ by the cell-like map of Keesling $[\mathrm{K}] g: Q \rightarrow Y$ onto 
an infinite dimensional compact metric space $Y$ such that $\tilde{K}^{*}(Y) \not \approx 0$, where $\tilde{K}^{*}$ denotes the reduced K-theory.

\section{REFERENCES}

[C-C] J. H. Case and R. E. Chamberlin, Characterizations of tree-like continua, Pacific J. Math. 10 (1960), 73 - 84. MR 22:1868

[D-K] J. Dydak and G. Kozlowski, Vietoris-Begle theorem and spectra, Proc. Amer. Math. Soc. 113 (1991), 587 - 592. MR 91m:55001

[K] J. E. Keesling, A non-movable trivial-shape decomposition of the Hilbert cube, Bull. Acad. Polon. Sci. Ser. Sci. Math. Astronom. Phys. 23 (1975), 997 - 998. MR 52:11922

[Ma] S. Mardešić, A non-movable compacta with movable suspension, Bull. Acad. Polon. Sci. Sér. Sci. Math. Astronom. Phys. 19 (1971), 1101 - 1103. MR 46:6325

[Ma-S] S. Mardešić and J. Segal, Shape Theory, North-Holland Publishing Company, 1982. MR 84b:55020

[Mi] T. Miyata, Generalized stable shape and duality, Topology Appl. 109 (2001), 75 - 88. CMP 2001:06

[Mi-S] T. Miyata and J. Segal, Generalized stable shape and the Whitehead theorem, Top. and its Appl. 63(1995), 139 - 164. MR 96d:54017

Department of Computer Science, Shizuoka Institute of Science and Technology, 2200-2 Toyosawa, Fukuroi, 437-8555 JaPan

Current address: Division of Mathematics and Informatics, Department of Science of Human Environment, Faculty of Human Development, Kobe University, 3-11 Tsurukabuto, Nada-Ku, Kobe 657-8501, Japan

E-mail address: tmiyata@kobe-u.ac.jp

Department of Mathematics, Faculty of Education, Yamaguchi University, YamAGUCHI, 753 JAPAN

E-mail address: tadashi@po.yb.cc.yamaguchi.ac.jp 